\title{
Sedoanalgesia en neonato a base de remifentanyl. En relación con un caso para epiglotopexia
}

\section{Sedoanalgesia in neonate based on remifentanyl. In relation to a case for epiglottopexy}

Dra. María Mayela Madriz-Godoy, ${ }^{*}$ Dr. Eloy Sánchez-Hernández, ${ }^{\ddagger}$ Dr. Enrique Gerardo Ortiz-Hernández ${ }^{\S}$

\begin{abstract}
RESUMEN. La etapa neonatal comprende del primer día hasta el día 28 de vida. Se caracteriza por ser una etapa de inmadurez anatómica, fisiológica y metabólica. Por estas características del neonato, los fármacos anestésicos impactan de manera importante en su fisiología respiratoria y cardiovascular; además la farmacocinética se ve modificada por esta inmadurez. La laringomalacia es una de las principales causas de estridor en esta etapa, misma que, dependiendo de la severidad puede requerir manejo quirúrgico, siendo una opción la epiglotopexia. Dicho procedimiento requiere analgesia potente y a su vez la posibilidad de mantener la ventilación espontánea para su adecuada evaluación, mismas que pueden ser alcanzadas con el uso de remifentanyl. Presentamos el caso clínico de un neonato de término de 22 días de vida a quien se le administró remifentanyl como base de la sedoanalgesia para realización de epiglotopexia de manera exitosa. Su potencia analgésica, capacidad de mantener el automatismo ventilatorio y la farmacocinética predecible en el neonato, convierten al remifentanyl en un agente ideal para proporcionar adecuada sedoanalgesia para diferentes procedimientos con un perfil de fácil titulación y de recuperación rápida en el neonato.
\end{abstract}

ABSTRACT. The neonatal stage comprises from the first day of birth until day 28 of life. It is characterized by being a stage of anatomical, physiological and metabolic immaturity. Due to these characteristics of neonates, anesthetic drugs have an important impact on their respiratory and cardiovascular physiology, and their immaturity on pharmacokinetics. Laryngomalacia is one of the main causes of stridor in this stage, which depending on its severity could require surgical treatment, such as an epiglotopexy. This procedure requires potent analgesia and at the same time the possibility of maintaining spontaneous ventilation for its appropriate evaluation. This can be achieved with the use of remifentanyl. We present the clinical case of a term neonate of 22 days of age who we administered Remifentanyl as the basis of sedoanalgesia for the successful completion of an epiglotopexy. Its analgesic potency, ability to maintain ventilatory automatism and predictable pharmacokinetics in the neonate, makes it an ideal agent to provide adecuate sedoanalgesia for different procedures with an easy titration profile and rapid recovery in the neonate.

Abreviaturas:

ASA = Clasificación del estado físico de la Sociedad Americana de

Anestesiólogos.

CAM =Concentración alveolar mínima.

$\mathrm{Cl}=$ Aclaramiento.

$\mathrm{CO}_{2}=$ Dióxido de carbono.

$\mathrm{Cp}=$ Concentración plasmática.

CPAP = Presión Positiva continua en las vías respiratorias.

FDA = Food and Drug Administration.

$\mathrm{FiO}_{2}=$ Fracción inspirada de oxígeno.

$\mathrm{PBE}=$ Peso bajo para la edad.

PBEG = Peso bajo para la edad gestacional.

$\mathrm{PEEP}=$ Presión positiva al final de la expiración.

PIP = Presión inspiratoria pico.

PSV = Ventilación con presión de soporte.

$\mathrm{Rpm}=$ Respiraciones por minuto .

SDG $=$ Semanas de gestación .

SIMV = Ventilación mandatoria intermitente sincronizada.

UCIN = Unidad de Cuidados Intensivos Neonatales.
Anestesiologla

Enero-Marzo 2020

Vol. 43. No. 1. pp 64-68

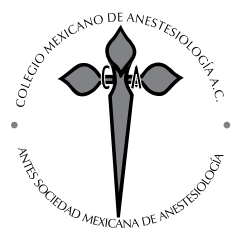

${ }^{*}$ Residente de segundo año en Anestesiología Pediátrica.

‡ Anestesiólogo Pediatra, adscrito al Servicio de Anestesiología. Profesor Titular del Curso de Anestesiología Pediátrica.

$\S$ Otorrinolaringólogo Pediatra, adscrito al Servicio de Otorrinolaringología y Cirugía de Cabeza y Cuello.

OPD Hospital Civil de Guadalajara «Fray Antonio Alcalde».

\section{Palabras clave:}

Remifentanyl, neonato, recién nacido, epiglotopexia, sedoanalgesia, anestesia.

\section{Keywords:}

Remifentanyl, neonate, newborn, epiglottopexy, sedoanalgesia, anesthesia.

Solicitud de sobretiros: Dr. Eloy Sánchez-Hernández OPD Hospital Civil de Guadalajara

«Fray Antonio Alcalde». Calle Hospital Núm. 278, Col. El Retiro, Guadalajara, Jalisco, México.

Tel: +52 39424400, ext. 41045

E-mail: dreloy124@hotmail.com

Recibido para publicación: 28-06-2018

Aceptado para publicación: 31-10-2018 


\section{INTRODUCCIÓN}

L a etapa neonatal comprende del primer día hasta el día 28 de vida. Este grupo etario se caracteriza por presentar inmadurez de los diferentes sistemas orgánicos, enzimático, así como múltiples diferencias anatómicas. Las características de su vía aérea en desarrollo e inmadurez del centro respiratorio los hacen propensos a períodos de apnea y colapso a nivel pulmonar $^{(1,2)}$. Estas características convierten a los neonatos en una población vulnerable a todas las intervenciones, ya sea de tipo mecánicas o farmacológicas a nivel respiratorio.

Dentro de estas diferencias anatómicas la presencia de una epiglotis más larga favorece su desplazamiento posterior. Esto, aunado a cartílagos más laxos comparados con la edad adulta, lo predisponen a una mayor colapsabilidad de la vía aérea. La laringomalacia es la anomalía laríngea más común y es la principal causa de estridor (60-70\% de los casos en neonatos y lactantes), presentándose de manera habitual en las primeras dos semanas de vida. Ante la sospecha, el diagnóstico se confirma bajo fibrolaringoscopía y se observa la dinámica laríngea bajo respiración espontánea. Los síntomas van de estridor leve hasta colapso de la vía aérea con riesgo de asfixia, situación que amenaza la vida. Es por esto que, si la severidad de los síntomas lo amerita, se puede requerir intervención quirúrgica. Previo al desarrollo de técnicas como la epiglotopexia, la traqueostomía era el estándar de oro para garantizar una adecuada dinámica respiratoria, asegurando la vía aérea posterior a la obstrucción laríngea; sin embargo, tiene numerosos riesgos y complicaciones, sobre todo en la población pediátrica ${ }^{(3)}$.

La epiglotopexia es uno de los manejos existentes para el prolapso obstructivo epiglótico, consiste en retirar parte de la mucosa de la valécula y de la base de la lengua utilizando electrocauterio o láser de $\mathrm{CO}_{2}$, suturando la epiglotis a la base de la lengua bajo visión endoscópica ${ }^{(4)}$. Durante el procedimiento es aconsejable mantener al paciente con ventilación espontánea para la valoración dinámica de la respiración y la movilidad de las estructuras. Lo anterior, sumado a las características anatomofisiológicas del neonato, nos obliga a planear nuestro manejo anestésico en el cual debemos considerar el grado de inmadurez hepática, renal, cardiovascular, respiratoria, sistema nervioso central, así como de los sistemas enzimáticos y de unión a las proteínas, que impactan la farmacocinética en este grupo ${ }^{(5,6)}$. A pesar de que el sistema nervioso central no está desarrollado en su totalidad al nacimiento, es de vital importancia considerar que las vías del dolor están integradas desde momentos tempranos en la gestación ${ }^{(6)}$.

Dentro de las opciones farmacológicas con las que contamos están los opioides, y de éstos, los de vida media corta, rápida eliminación y metabolismo por esterasas plasmáticas como el remifentanyl; éste es una buena opción en el neonato, ya que las esterasas están presentes al nacimiento, por lo que se puede predecir su aclaramiento a pesar de las diferencias anatomofisiológicas importantes en el neonato ${ }^{(5,7,8)}$.

Presentamos el caso de un neonato de 22 días de vida con laringomalacia y múltiples intentos fallidos de extubación, el cual se programa para realización de laringoscopía diagnóstica y realización de epiglotopexia.

\section{CASO CLÍNICO}

Paciente femenino de 22 días de vida extrauterina, peso 2,800 g, talla $49 \mathrm{~cm}$. Diagnóstico de laringomalacia con cuatro intentos de extubación fallida. Antecedentes perinatales: madre de 23 años quien inició control prenatal en primer trimestre con consumo de hematínicos. Durante el embarazo se le diagnosticó holoprosencefalia mediante ecografía. Se obtuvo vía vaginal a las 38.2 SDG con circular de cordón apretada, Apgar al minuto de siete. Requirió apoyo con presión positiva e intubación orotraqueal por 15 minutos. Se extubó y se mantuvo con CPAP nasal, ingresa a la UCIN por síndrome de dificultad respiratoria y estridor laríngeo. Peso al nacer 2,345 g PBEG. Requirió intubación orotraqueal a su ingreso a la UCIN el primer día de vida; 24 horas después se intentó extubar sin éxito. En tres ocasiones más se extubó la tráquea presentando en todas las ocasiones estridor laríngeo posterior a ello, con datos de dificultad respiratoria, por lo que se reintubó y mantuvo bajo ventilación mecánica con SIMV (30 rpm, PIP 12, PEEP 5, PSV 9, $\mathrm{FiO}_{2} 30 \%$ ). Hemodinámicamente estable. Laboratorialmente con hemoglobina de $10.9 \mathrm{~g} / \mathrm{dL}$, hematocrito $32.2 \%$ y plaquetas 554,000 por $\mu \mathrm{l}$. Se transfundió 10 mililitros por kilo de peso previo al procedimiento quirúrgico. Neurología no relaciona la dificultad para la extubación con patología de sistema nervioso central. Es valorada como ASA III, se programó para laringoscopía diagnóstica y epiglotopexia. El planteamiento anestésico es una sedoanalgesia a base de remifentanyl y sevoflurano.

La paciente ingresó a sala de procedimientos, despierta, orointubada con cánula 3.5, sin infusión de fármacos hipnóticos-sedantes y/o analgésicos previos. Se monitorizó de forma no invasiva con electrocardiograma de tres electrodos y oximetría de pulso. Se continuó con el plan anestésico. En una vía periférica iniciamos infusión de remifentanyl en perfusor a una concentración plasmática objetivo de $0.004 \mu \mathrm{g} / \mathrm{mL}$, dicha dosis fue calculada con la fórmula de $\mathrm{Cp} \times \mathrm{Cl}(40) \mathrm{xg}$. Iniciamos administración de sevoflurano $1 \%$ con $\mathrm{FiO}_{2}$ al $100 \%$ y flujo de $3 \mathrm{~L} / \mathrm{min}$. Se mantuvo la ventilación espontánea y estabilidad hemodinámica. Se extubó y cinco minutos después inició el procedimiento, se introdujo fibroscopio flexible para evaluar la dinámica laríngea y características glóticas confirmándose el diagnóstico de laringomalacia (Figura 1). Se decidió realizar la epiglotopexia, para la cual se colocó el laringoscopio de suspensión de Kleinsasser y se utilizó el broncoscopio rígido 


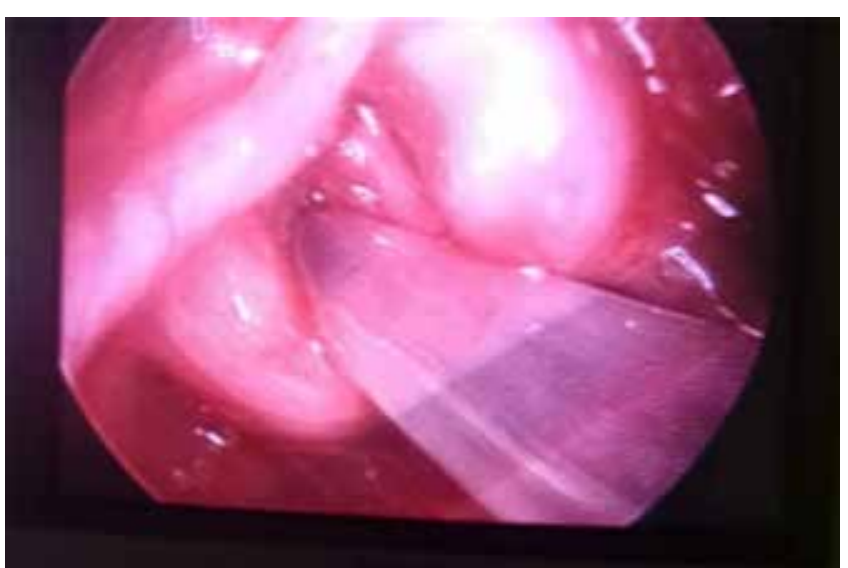

Figura 1: Evaluación inicial de la laringe. Diagnóstico de laringomalacia.

para realizar la epiglotopexia mediante puntos percutáneos en la cara anterior del cuello (Figura 2).

El procedimiento duró 40 minutos. Presentó un hematoma periepiglótico derecho con complicación de la técnica quirúrgica. La anestesia transcurrió sin eventualidades, con cambios hemodinámicos menores al 10\% de sus basales (Tabla 1). No hubo necesidad de realizar ajustes en el transanestésico a la infusión de remifentanyl ni del sevoflurano. Como adyuvantes administramos paracetamol $15 \mathrm{mg} / \mathrm{kg}$ y dexametasona $0.3 \mathrm{mg} / \mathrm{kg}$.

Una vez concluido el procedimiento se suspendió la administración de remifentanyl. Se decidió intubar nuevamente y se trasladó orointubada con ventilación espontánea, asistida con bolsa autoinflable a Unidad de Cuidados Intensivos Neonatales. Permaneció intubada en espera de resolución del hematoma y edema secundario al procedimiento, para después valorar extubación, misma que se logró a los tres días sin complicaciones.

\section{DISCUSIÓN}

El neonato con síntomas severos asociados a laringomalacia se convierte en candidato para manejo quirúrgico. Existen dos principales abordajes: la supraglotoplastía y la epiglotopexia ${ }^{(3,9)}$. Esta última consiste en fijar la epiglotis a la superficie lingual, en la base de la lengua ${ }^{(9)}$, por medio de la aplicación de puntos percutáneos desde la cara anterior del cuello, evitando de esta manera su colapso sobre la apertura glótica con consecuente obstrucción de la vía aérea. Para lograr una adecuada evaluación del grado de obstrucción pre, trans y postoperatoria es necesario mantener la ventilación espontánea para observar la dinámica laríngea bajo endoscopía ${ }^{(10)}$. Esto puede ser alcanzado con la elección de fármacos como el remifentanyl, de acción ultra corta y fácilmente titulable que atenúa la respuesta neuroadrenérgica a la laringoscopía e intubación orotraqueal, lo que brinda una analgesia adecuada, un excelente control intraoperatorio, la posibilidad de mantener la ventilación espontánea así como una óptima calidad de recuperación anestésica ${ }^{(11)}$.

El neonato puede presentar una respiración periódica y desaturación, incluso en ausencia de enfermedad, cirugía o procedimiento anestésico ${ }^{(12)}$, por lo que es esperada una sensibilidad incrementada al uso de anestésicos que tenemos disponibles por las características anatomofisiológicas mencionadas. Especialmente la inmadurez del centro respiratorio, el cual es, en parte, responsable del efecto pronunciado que tienen los diferentes anestésicos en esta población, siendo el grupo con mayor incidencia de apnea perioperatoria ${ }^{(6,7)}$. Es importante considerar que, en función de si existe actividad de la musculatura respiratoria, las apneas se pueden clasificar en centrales y obstructivas, ambas multifactoriales. En el caso del neonato con laringomalacia sometido a un procedimiento anestésico, la presentación de apnea tendría un componente mixto, por lo que el hecho de que el uso de opioides de acción intermedia o larga propicie un despertar prolongado e incluso la necesidad de ventilación mecánica postoperatoria, hace que se descarte su uso como fármaco de primera elección ${ }^{(6,13)}$.

Cabe mencionar que la característica cartilaginosa de la caja torácica de los neonatos les brinda una gran complianza; sin embargo, sumado a la presión intrapleural negativa mucho

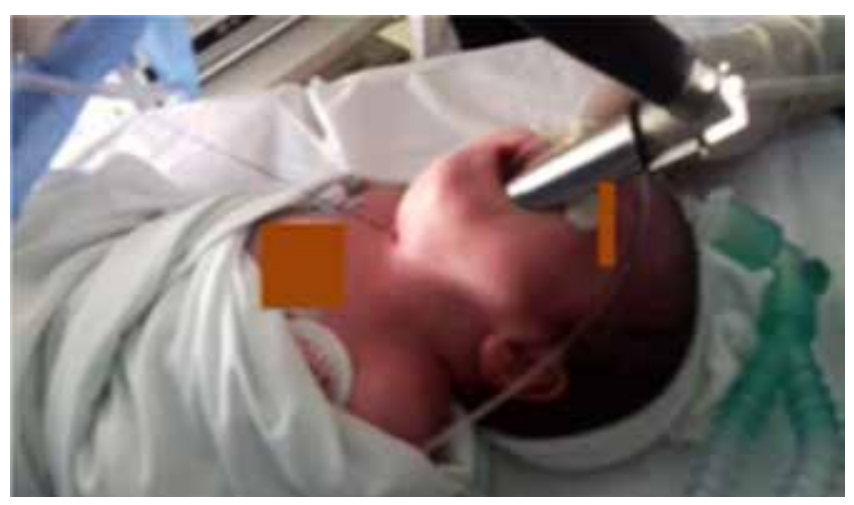

Figura 2: Colocación del laringoscopio de suspensión de Kleinsasser y punción percutánea para la epiglotopexia.

Tabla 1: Parámetros hemodinámicos transanestésicos.

\begin{tabular}{|c|c|c|c|c|c|}
\hline $\begin{array}{l}\text { Signos } \\
\text { vitales }\end{array}$ & Basales & $\begin{array}{l}10 \\
\min \end{array}$ & $\begin{array}{l}20 \\
\min \end{array}$ & $\begin{array}{l}30 \\
\min \end{array}$ & $\begin{array}{l}40 \\
\min \end{array}$ \\
\hline FC (lat/min) & 174 & 165 & 166 & 166 & 163 \\
\hline $\mathrm{SpO}_{2}(\%)$ & 98 & 96 & 97 & 97 & 98 \\
\hline
\end{tabular}


menor que el resto de la población, los hace propensos al colapso pulmonar. Ello condiciona a que deban incrementar la resistencia respiratoria a este colapso a través de mecanismos como la abducción laríngea, apoyo espiratorio por medio de activación de los músculos espiratorios y manteniendo frecuencias respiratorias elevadas para limitar el tiempo espiratorio. Además, debido al pequeño calibre de su vía aérea se presenta en ella hasta $80 \%$ de la resistencia al flujo de gas hasta los pulmones ${ }^{(1,2)}$.

El remifentanyl es un agonista opioide mu de acción ultracorta, rápido inicio de acción y efecto pico, aprobado por la FDA (U.S. Food \& Drug Administration) para su uso en el neonato, excepto en los menores de 29 SDG ${ }^{(14,15)}$. Alcanza su pico máximo de analgesia en el primer minuto de su administración, tres a cuatro veces más rápido que el fentanilo. Su metabolismo es por medio de esterasas plasmáticas y tisulares inespecíficas, mismas presentes y con actividad madura al nacimiento, por lo que su aclaramiento es predecible a pesar de la inmadurez del resto de los órganos ${ }^{(5,7,15)}$.

En cuanto a las dosis de infusión de remifentanyl, la recomendación por la FDA en el paciente menor de dos meses es de $0.4-1 \mu \mathrm{g} / \mathrm{kg} / \mathrm{min}$ balanceado con óxido nitroso. La recomendación en pacientes de 1-12 años es de 0.05-1.3 $\mu \mathrm{g} / \mathrm{kg} / \mathrm{min}$, cuando se balancea con sevoflurano de 0.3-1.5 $\mathrm{CAM}^{(15)}$. Con el empleo de estas dosis es posible mantener la ventilación espontánea y a la vez se brinda excelente analgesia para llevar a cabo procedimientos dolorosos. En este caso utilizamos una infusión de $0.44 \mu \mathrm{g} / \mathrm{kg} / \mathrm{min}(0.004 \mu \mathrm{g} /$ $\mathrm{mL}$ de concentración plasmática objetivo) acompañado de sevoflurano a 1\% (0.3 CAM). Realizaron la evaluación inicial con fibroscopía flexible bajo ventilación espontánea, posteriormente se hizo la laringoscopía y suspensión laríngea sin que se presentaran cambios hemodinámicos que nos alertaran de analgesia inadecuada.

Wee y colaboradores mostraron que un rango de 0.05-1 $\mu \mathrm{g} / \mathrm{kg} / \mathrm{min}$ de remifentanyl (eliminando dosis en bolo) es seguro para utilizar en neonatos, condicionando un tiempo de extubación de 21 min en menores de siete días y de seis minutos en mayores de siete días ${ }^{(16)}$. Por otra parte, Kessler y su equipo utilizaron una dosis media de $0.77 \mu \mathrm{g} / \mathrm{kg} / \mathrm{min}$ con un tiempo medio de extubación de 7.7 minutos en neonatos sometidos a broncoscopía con asociación de propofol/ mivacurio con excelentes resultados ${ }^{(17)}$. Davis y colegas compararon el uso de remifentanyl/óxido nitroso y halotano/ óxido nitroso en un grupo de neonatos y lactantes menores de ocho semanas. La dosis media utilizada de remifentanyl en infusión fue de $0.34 \mu \mathrm{g} / \mathrm{kg} / \mathrm{min}$, un tiempo de extubación promedio de 7.5 min en ambos grupos, sin casos de apnea postoperatoria en el grupo de remifentanyl frente a $23 \%$ en el grupo de halotano ${ }^{(18)}$. Barker y su grupo concluyeron que los pacientes menores a tres años toleran infusiones de $0.35 \mu \mathrm{g} / \mathrm{kg} / \mathrm{min}$ con adecuada ventilación espontánea con o sin adición de algún agente halogenado, como nuestro caso presentado $^{(19,20)}$.

Un punto de relevancia en la farmacocinética del neonato es el aclaramiento. Dicha variable para el remifentanyl en pacientes de cinco días de vida hasta los 17 años es igual que en el adulto. Esto, aunado a su rápida lisis enzimática, determina que en cinco a 10 minutos de que se suspende su administración intravenosa se tenga recuperación completa de sus efectos, tanto ventilatorios como analgésicos, es por ello que la analgesia para el postoperatorio debe estar cubierta al término de su administración ${ }^{(5,15)}$. Para su manejo nos podemos apoyar en la administración de diferentes fármacos, entre los que se encuentran los antiinflamatorios esteroideos y no esteroideos, infiltración local, bloqueos regionales, ketamina, lidocaína y sulfato de magnesio por mencionar algunos. En este caso utilizamos dexametasona como antiinflamatorio y paracetamol como analgésico, sin necesidad de agregar otros adyuvantes.

\section{CONCLUSIÓN}

Concluimos que el remifentanyl debido a su rápido inicio de acción, metabolismo por esterasas, potencia analgésica y facilidad para titulación en el paciente neonato lo convierten en un excelente agente para manejo anestésico en este grupo etario, ya que a pesar de tener inmadurez de múltiples vías metabólicas, las esterasas plasmáticas y tisulares están presentes y maduras al nacer, garantizando su total aclaramiento. Todas estas características lo convierten en un agente ideal para proporcionar sedoanalgesia en diferentes procedimientos con un perfil de fácil titulación y de recuperación rápida en el neonato y que además nos permite mantener la ventilación espontánea en caso de así requerirse ${ }^{(7,8,20)}$. 


\section{REFERENCIAS}

1. Davis R, Mychaliska G. Neonatal pulmonary physiology. Semin Pediatr Surg. 2013;22:179-184.

2. Neumann RP, Von Ungern-Sternberg BS. The neonatal lung-Physiology and ventilation. Paediatr Anaesth. 2014;24:10-21.

3. Dobbie AM, White DR. Laryngomalacia. Pediatr Clin North Am. 2013;60:893-902.

4. Colaianni C, Bowe S, Osborn H, Lin D, Richmon J, Hartnick C. Robotic epiglottopexy for severe epiglottic prolapse limiting decannulation. Int J Pediatr Otorhinolaryngol. 2017;102:157-159.

5. Allegaert K, Van den Anker J. Neonatal pain management: still in search of the holy grail. Int J Clin Pharmacol Ther. 2016;54:514-523.

6. Martin-Lynn D. The basic principies of anesthesia for the neonate. Rev Colomb Anestesiol. 2017;45:54-61.

7. Kamata M, Tobias J. Remifentanyl: application in neonates. J Anesth. 2016;30:449-460.

8. Hernández-Gutiérrez D, Sánchez-Hernández E, Ramírez-Aldana L, García-Arreola DAP. Remifentanyl en pediatría. Diferentes escenarios de aplicación. Rev Mex Anest. 2012;35:168-171.

9. Werner J, Lippert B, Dunne A, Ankermann T, Folz B, Seyberth $H$. Epiglottopexy for the treatment of severa laryngomalacia. Eur Arch Otorhinolaryngol. 2002;259:459-464.

10. Oomen K, Modi V. Epiglottopexy with and without lingual tonsillectomy. Laryngoscope. 2014;124:1019-1022.

11. Reinoso F, Castro L. Influencia de la edad pediátrica en la técnica de anestesia basada en la analgesia con remifentanilo. Rev Esp Anestesiol Reanim. 2004;51:12-19.
12. Rothstein P. Remifentanyl for neonates and infants: piano, piano con calma. Anesth Analg. 2001;93:1370-1372.

13. American Heart Association. Soporte vital avanzado pediátrico libro del proveedor. EUA; 2017.

14. Bang SR. Neonatal anesthesia: how we manage our most vulnerable patients. Korean J Anesthesiol. 2015;68:434-441.

15. FDA. U.S. Food \& Drug Administration. [Online]. Disponible en: https:// www.accessdata.fda.gov/drugsatfda_docs/label/2004/20630se5-005_ ultiva_lbl.pdf.

16. Wee LH, Moriarty A, Cranston A, Bagshaw O. Remifentanil infusion for major abdominal surgery in small infants. Paediatr Anaesth. 1999;9:415418.

17. Kessler P, Ahrens P, Lischke V, et al. Einsatz von Remifentanyl bei Neugeborenen und Sauglingen. Anaesthesiologie und Intensivmedizin. 1999;40:Abstractband DAK 99.

18. Davis PJ, Galinkin J, McGowan FX, et al. A randomized multicenter study of remifentanil compared with halothane in neonates and infants undergoing pyloromyotomy. I. Emergence and recovery profiles. Anesth Analg. 2001;93:1380-1386, table of contents.

19. Barker N, Lim J, Amari E, Malherbe S, Ansermino JM. Relationship between age and spontaneous ventilation during intravenous anesthesia in children. Pediatr Anesth. 2007;17:948-955.

20. Sammartino M, Garra R, Sbaraglia F, De Riso M, Continolo N. Remifentanyl in children. Paediatr Anaesth. 2010;20:246-255. 